\title{
Game Theory-Based Routing Algorithms for Wireless Multi-hop Networks
}

\author{
Zhi Ren, Shuang Peng, Hongjiang Lei, Jibi Li \\ Chongqing Key Lab of Mobile Communications Technology, Chongqing University of Posts and \\ Telecommunications Chongqing, China 400065 \\ Email: renzhi@cqupt.edu.cn
}

\begin{abstract}
In wireless multi-hop networks, the quality of service provided by the system heavily depends on the cooperation between nodes. However, nodes are reluctant to cooperate with each other to save their limited resources. This tendency will put a limit on the application of the current wireless multi-hop networks. Therefore, game theory, which can analyze the strategic interactions among autonomous decision makers, becomes an ideal candidate to solve the above problem. In this paper, we first introduce the basic concept of game theory. Then, the game-theory-based routing algorithms for wireless multi-hop networks are investigated in detail and their characteristics are compared. Finally, we give the future research directions and concluding remarks.
\end{abstract}

Keywords-Wireless multi-hop networks; Routing algorithms; Game theory; Cooperation

\section{INTRODUCTION}

In wireless multi-hop networks, the communication between nodes must rely on the collaboration of relay for the mobility of nodes, the distribution of the network and so on. Therefore, the quality of the service provided by the system heavily depends on the cooperation between nodes. However, the nodes are reluctant to delivery packets for other nodes to save their limited resources. These inevitably affect the performance of network. Now, the proposed solutions to stimulate cooperation can be generally categorized three solutions: (1) virtual currency based methods [1-2], (2) reputation based mechanisms [3-4], (3) game theory mechanisms [5-6](the most promising approaches).

Game theory is the most appropriate mechanism to model, analyze and solve the problems of stimulating selfish nodes to cooperate. The reasons are as follows, first, nodes are rational agents which making decisions only for their own interests. Second, game theory can provide an efficient framework to deal with distributed optimization only using local information. Finally, game theory designs mechanisms which intermediate nodes can sell their resources to the source nodes to assist forwarding. Therefore, Game theory is an ideal candidate to realize the cooperation between nodes. In this paper, we will present several routing algorithms based on game theory and make a comparison between them. Then, we will narrative the reference to the wireless multi-hop networks.

The rest of this paper is organized as follows: In section II, we describe the architecture of game theory and routing algorithms. In section III, we classify the current routing algorithms based on game theory and illustrate their respective basic principles. In section IV, we compare and analyze the performance of the present routing algorithms. Section V conclusion and future works are given.

\section{ARCHITECTURE AND ROUTING MECHANISMS}

\section{A. Basic application of game theory}

Game theory is a tool aimed at analyzing the strategic interactions among autonomous decision makers, whose actions have mutual, probably conflicting, consequences. It is widely used in the economic world where people interact with each other. For example, Auction is a decentralized market mechanism for allocating resources. Originally developed to model problems in the field of economics, game theory has recently been applied to network problems, in most cases to stimulating selfish nodes to cooperate.

\section{B. Basic concepts of game theory}

A game consists of three major components [11, 13]:

- Players: The decision makers are called players, denoted by a finite set $N=\{1,2, \ldots, n\}$.

- Strategy: Each player $i \in N$, has a non-empty strategy set $S_{i}$. Let $s_{i}$ denote the selected strategy by player i. A strategy profile s consists of all players' strategies, i.e., $\mathrm{s}=\left(s_{1}, s_{2}, \ldots, s_{n}\right)$. Obviously, we have $s_{i} \in S=\times_{i \in N} S_{i}$, where $\times$ is the Cartesian product.

- Utility/payoff: The utility of player $i$ is a measurement function, denoted by $u_{i}, S \rightarrow R$, on the possible outcome determined by the strategies of all players, where $\mathrm{R}$ is the set of real numbers.

\section{ROUTING ALGORITHMS BASED ON GAME THEORY}

Game theory has been used in many routing algorithm design (such as energy optimization, power control, network capacity, stability, etc.). According to different principles, routing algorithms based on game theory can be divided into non-cooperative game-based routing algorithm and cooperative game-based routing algorithm[12], noncooperative game can be divided into recourse energy and power control[17], cooperative game can be divided into cluster routing and forward strategy, as shown in Figure 1. 


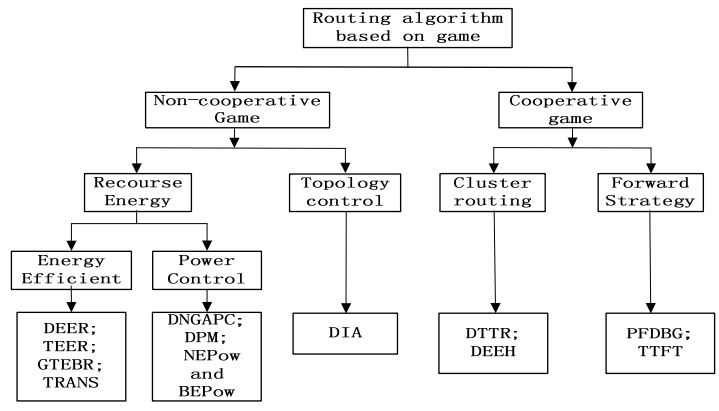

Figure 1. Taxonomy of routing algorithms based on Game Theory

\section{A. Cooperative game clustering routing-Based algorithm}

1) DTTR [9]: The algorithm adopts the basic principle of LEACH algorithm, but improved. DTTR uses the same set of clustering algorithm and omits the re-clustering and cluster head rotation. The main principle of clustering management strategy of DTTR is described as follows: DTTR establishes a network model based on game theory including the participants collection of $\mathrm{S}$, the pure strategy set $\mathrm{L}$ and payoff function set $\pi$. The intermediate node is selected based on the value of $\pi$, then nodes exclude those neighbor nodes below a certain threshold in order to keep the link quality. After that, neighbor nodes are divided into two groups based on neighbor distance away from the gateway, one group distance from the gateway is less than the node, the other is greater or equal to the node. Then, based on the value of $\pi$ calculate by DTTR ranks neighbor nodes, and select each group maximum value node as the next hop node of the two kinds of data flows. If receipt data packet, the node select the next hop node according to its destination address. If not, the node immediately selects maximum value of $\pi$ from the sort queue to continue sending the data packet. DTTR can improve throughput and reduce the standard deviation of residual energy. However, it is difficult to set the estimation value of the link forwarding probability.

2) DEEH[4]: The DEEH Routing algorithm, an enhanced version of LEACH clustering routing algorithm. Comparing with classical clustering LEACH algorithm with algorithm, DEEH performance is better when network node density is large, which considers the existence of selfish nodes and the impact of a selfish node network clustering in the network. DEEH calculates the expected delay adopts game theory algorithm to the dominant strategy for selfish nodes truthfully report their energy. When the utility value is distributed appropriately in the network, DEEH can prolong the life of the network to ensure the stability of the topology.

\section{B. Cooperative game forwarding strategy-Based routing algorithm}

1) PFDBG[10]: PFDBG is a repeat game with incomplete information and observable actions. The main principle of PFDBG algorithm is described as follows: each node puts its interference power value into HELLO messages and broadcast to its neighborhood nodes, then calculates its income and update its adjacent node information table according to the algorithm when receive a HELLO packet. After that, if a node needs to send packets, it determines its required transmit power value based on adjacent node information table in PFDBG algorithm. PFDBG algorithm can ensure higher throughput and low energy consumption in multi-hop networks.

2) TTFT[15]: TTFT is a fault-tolerant tit-for-tat strategy TFT[21]. Under TTFT strategy, each node always chooses to cooperate until the first time slot of other nodes behave selfishly. The selfish node will be punished for $\mathrm{n}$ time slots unconditional cooperation, which is called tolerance stage. If there are $\mathrm{M}$ slots non-cooperation within $\mathrm{N}$ time slots in the tolerance stage, its neighbor nodes start from the $(n+1)$ th slot taking tit-for-tat strategy, which is called inspection stage. After the end of the $\mathrm{m}^{*} \mathrm{n}$ slots tit-for-tat strategy, the selfish node restart behavior is no longer considered. In the fault case of network connections, the TTFT scheme can show a better performance. Nodes tend to adopt a cooperative behavior when they find the payoff of selfish node is less than the average yield of the cooperation nodes.

\section{Non-cooperation game energy saving-Based routing algorithm}

1) DEER [18]: DEER is based on non-cooperation game theory. In DEER, the cluster head node payoff function is $\pi$, each node establish neighbor information table and broadcast value of $\pi$. When receiving adjacent nodes $\pi$ value, node compares its own $\pi$ and selects larger value of $\pi$ recorded in the neighbor information table. If adjacent node information set is empty, nodes automatically become a cluster head and broadcast cluster head selection information. When receiving one or more cluster head selection information, node sends attribution information which added value of $\pi$ to the largest cluster head node. Meanwhile, under multiple cluster head selecting, if several cluster head nodes with the same value of $\pi$, node random select a cluster as vested send attribution information. After all participant nodes deciding their own strategies and building hierarchical routing, nodes start data transfer. In the worst case of network connections, DEER can effectively make the cluster head node uniform rational distribution and balance network load.

2) GTEBR [8]: GTEBR is a game model based on energy balance. In GTEBR algorithm, the probability of node selected as relay nodes is $\mathrm{p}$. The probability $\mathrm{p}$ is influenced by the node's residual energy, the past decision-making and action of a node, and so on. Then, the income is large if only one node forwarding data. If not, the income is small relatively. Moreover, the algorithm designs a suitable solver mechanism which makes the data forwarding area reach the $\mathrm{NE}$, thus the forward path distribution of the network becomes more uniformly. However, the link-state information in the process of exchanging will consume redundantly network bandwidth and nodal resources. 


\section{Non-cooperation game-Based power control routing algorithm}

1) DNGAPC[14]: DNGAPC is a distributed noncooperation power control game algorithm. The node calculates its transmission power based on the received HELLO packet broadcast by the neighbor nodes which include adjacent node information table. Meanwhile, this node update the adjacent nodes information table. Thus a node will search the adjacent node information table and calculate the transmission power when this node needs to send packets. Therefore, the DNGAPC algorithm minimizes the delay of broadcast and obviously improves the energy efficiency of message sending.

2) NEPow and BEPow [20]: NEPow is a NE power control algorithm and BEPow is a Bayesian NE power control algorithm. In NEPow and BEPow, there are different types of nodes, and the node has different level of transmission power. When a node finds adjacent node congestion, this node will improve the power to find other adjacent nodes. The NEPow and BEPow algorithm can maintain reliable topology connectivity and reduce power consumption.

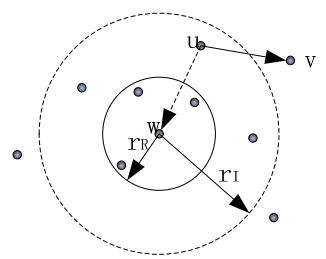

Figure 2. Interference model between network nodes

3) As shown in Figure 2, in the wireless multi-hop network, the node $\mathrm{w}, \mathrm{v}, \mathrm{u}$ randomly move. Thus, the distances and power can randomly change among them. The node $\mathrm{w}$ will increase interference with $\mathrm{u}$ and $\mathrm{v}$, if its randomly chose transmission power improves. Therefore, node $\mathrm{u}$ and $\mathrm{v}$ need to use a higher transmission power to overcome the influence of node $\mathrm{w}$. This caused the energy consumption of the network and decline network throughput. On the basis of the abovementioned features of this algorithm, Shamik Sengupta[22] proposed a new noncooperation incomplete information distributed optimal power control algorithm. This algorithm can make the node sends the minimum threshold power, reduce neighbor interference and save their energy consumption.

\section{E. Non-cooperation game-Based topology control routing algorithm}

1) TC[19]: In this algorithm, we assume each cluster head have the same initial transmission power and broadcast their remaining energy and ID message to the neighbor nodes. The neighbor nodes shall statistics received response message and calculates the average residual energy value after receiving the message. The algorithm can ensure network connectivity under the premise, thereby it can construct a more effective network topology control set.

2) DIA[7]: DIA is a routing algorithm which based on distributed non-cooperation power control. In DIA routing algorithm, each node obtains its neighborhood information by broadcasting "hello" beacon messages and replaying ACK at a certain power level. The hello messages should at least include the node's identification, current transmission power, and maximal transmission power. Thus, the DIA algorithm can ensure convergence to high efficiency energy consumption and topology connectivity.

\section{THE COMPREHENSIVE COMPARISON OF THE ROUTING ALGORITHMS BASED ON GAME THEORY}

In this section, we conduct a comparison with above routing algorithms analyze their feature in terms of cooperation/non-cooperation, link estimation and encounter probability, as shown in Table 1.

\section{A. Similarities of routing algorithms}

In order to suit the characteristics of node selfishness, several new mechanisms [11-12] based on game theory are designed in routing algorithms for multi-hop networks. In details, the repeated game scheme with parameter $(\mathrm{V}, \mathrm{T})$, for all users is explained as follows: Each user's utility $U$ is compared with the threshold $\mathrm{V}$. If $\mathrm{U}<\mathrm{V}$, i.e., someone deviates, the time counter $\mathrm{n}$ is set to zero, punish time is increased by one, and the user plays non-cooperatively for time of $\mathrm{T}$. We assume all users are rational, with increasing of $\mathrm{T}$, the benefit of one time deviation will be eliminated out sooner or later. Finally, no user wants to deviate and $U \geq V$. At this time, the counter $n$ starts increasing. If the system is stable in the cooperation for a period of time $\mathrm{N}$, where $\mathrm{N}$ is prudence constant, the algorithm assumes that the cooperation is enforced, and changes to the next step to improve the current cooperation $[14,16]$.

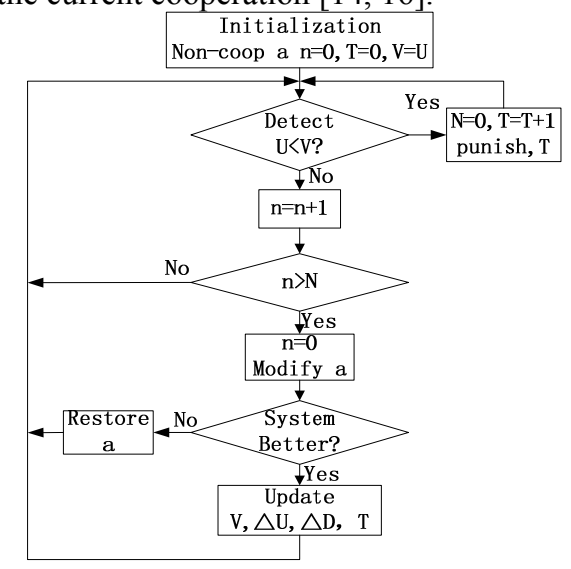

Figure 3. Architecture of algorithm process based on game theory

\section{B. Difference of routing algorithms}

We can see that each kind of routing algorithm has its advantages and drawback in terms of network efficiency and resources required. Cooperation is captured in terms of the node's probability to drop a message copy upon reception and/or to forward the message copy upon node encounter. The routing algorithms based on encounter probability can reduce effectively delivery of redundancy message and routing overhead. But node adopts historical information to estimate probability that may not match with the actual situation and affect packet delivery rate. The 
routing algorithm based on link forwarding probability also can reduce efficiently routing overhead and enhance the delivery rate. However, link state information's exchange and calculation will consume network bandwidth and node resources. The purpose of encounter probability and link forwarding probability is both to select a suitable next hop delivery node, which can effectively control the number of copies of data injected to the network and reduce network's communication cost. But this method will bring a potential risk which lead to increase of loss packet rates.

TABLE I. COMPARISON ON CHARACTERISTICS OF ROUTING ALGORITHMS

\begin{tabular}{|c|c|c|c|c|}
\hline $\begin{array}{c}\text { Routing } \\
\text { algorithm }\end{array}$ & $\begin{array}{c}\text { Cooperative/ } \\
\text { Non- } \\
\text { cooperative }\end{array}$ & $\begin{array}{c}\text { Estimation of } \\
\text { link forwarding } \\
\text { probability }\end{array}$ & $\begin{array}{l}\text { Reactive/ } \\
\text { Proactive }\end{array}$ & $\begin{array}{c}\text { Encounter } \\
\text { Probability }\end{array}$ \\
\hline DTTR & Yes & Yes & Proactive & No \\
\hline DEEH & Yes & No & Reactive & No \\
\hline PFDBG & Yes & Yes & Reactive & No \\
\hline TTFT & Yes & No & Reactive & Yes \\
\hline DEER & No & No & Reactive & No \\
\hline TEER & No & No & Reactive & Yes \\
\hline GTEBR & No & Yes & Reactive & No \\
\hline TRANS & No & No & Reactive & Yes \\
\hline DNGAPC & No & No & Proactive & Yes \\
\hline DPM & No & No & Reactive & Yes \\
\hline NEPow and EPow & No & No & Reactive & Yes \\
\hline DIA & No & No & Reactive & Yes \\
\hline
\end{tabular}

\section{CONCLUSIONS AND FUTURE DiRECTIONS}

The focus of this paper is to explore the impact of node selfish on some representative routing algorithms for wireless multi-hop network routing algorithm based on game theory. Although these algorithms solve partly of the node selfishness problem, there still exist a lot of critical issues such as the complexity of the network model, additional overhead, and node collusion hazards, although several node selfishness problems were solved by these algorithms. Therefore, in the future, we will further our study to consider solving the complexity of the networks model through dynamic game. Then, we will design optimize routing algorithm based on game theory to reduce the overhead.

\section{ACKNOWLEDGMENTS}

This work was supported in part by National Natural Science Foundation of China under Grant No. 60972068, the Scientific Research Starting Foundation for Returned Overseas Chinese Scholars, Ministry of Education of China under Grant No. 2010-1561, the Science Research Project of Education Committee of Chongqing under Grant No. $\mathrm{KJ} 120510$, and the transformation project of excellent achievement of Chongqing Municipal Education Commission under Grant No. Kjzh11206.

\section{REFERENCES}

[1] S. zhong, J. Chen, Y. Yang. "Sprite: A Simple, Cheat-Proof, CreditBased System for Mobile Ad-Hoc Networks," Proc. Computer and Communications (Infocom 22nd). IEEE Societies, Feb. 2003, pp. 1987-1997, doi:10.1109/INFCOM.2003.1209220.

[2] V. Srinivasan, P. Nuggehalli and C.F. Chiasseriniand. "Cooperation in Wireless Ad Hoc Networks," Proc. Computer and Communications (Infocom 22nd). IEEE Societies, April. 2003, pp. 807-817, doi:10.1109/INFCOM.2003.1208918

[3] S. Marti, T. J. Giuli and K. Lai. "Mitigating routing misbehavior in mobile ad hoc networks," Proc. ACM Symp. Mobile computing and networking (MobiCom 6th), ACM Press, Feb. 2000, pp. 255-265, doi: $10.1145 / 345910.345955$.

[4] W.D. Wang and Q.X. Zhu. "A Hierarchical Clustering Algorithm and Cooperation Analysis for Wireless Sensor Network," Journal of Software, vol. 17, May. 2006, pp. 1157-1167, doi:cnki:ISSN:10009825.0.2006-05-023.

[5] L.A. Dasilva, H. Bogucka and A.B. Mackenzie. "Game Theory in Wireless Networks," Communications Magazine. Vol. 49, August. 2011, pp. 110-111, doi:10.1109/MCOM.2011.5978423.

[6] L. Yan. "Cooperative Packet Relaying in Wireless Multi-hop Networks," Pro. IEEE Symp. Advanced Information Networking and applications Workshops. IEEE Press, August. 2009, pp. 345-350, doi:10.1109/WAINA.2009.11.

[7] R.S. Komali, A.B. MacKenzie and R.P. Gilles. "Effect of Selfish Node Behavior on Efficient Topology Design," IEEE Trans. Blacksburg, VA, vol. 7, Sept. 2008, pp. 1057-1070, doi:10.1109/TMC.2008.17.

[8] Zeng Jia, C.D. Mu and Jiang Min. "Game Theoretic Distributed Energy Control in Sensor Networks," Computer and Information Technology (CIT 7th). IEEE Press, Oct. 2007, pp. 1015-1019, doi:10.1109/CIT.2007.51.

[9] J. Hu, L.F. Shen. "Clustering routing protocol of wireless sensor networks based on game theory," International Workshop on Localized Algorithms and Protocols for Wireless Sensor Networks. Vol. 40, May. 2009, pp. 441-445, doi:10.3969/j.issn.10010505.2010.03.001.

[10] Y.H. Zhao, H.S. Shi. "Game theoretical packet forwarding algorithm in wireless sensor networks," Journal of Xidian University, May. 2010, vol 37, pp. 1125-1131, doi: CNKI: SUN:XDKD.0.2010-06-028.

[11] D.J. Yang, X. Fang and L.X. Guo. "Game Theory in Coperative Communications," IEEE Wireless Communications. Vol. 19, April. 2012, pp. 44-49, doi:10.1109/MWC.2012.6189412.

[12] W. Zhuang, M. Ismail. "Cooperation in Wireless Communication Networks,” IEEE Wireless Communications. Vol. 19, April. 2012, pp. 10-20, doi:10.1109/MWC.2012.6189408.

[13] D. Fudenberg and J. Tirole, Game Theory. MIT Press, 1991.

[14] Q. Sun, X.W. Zeng and N.S. Chen. "A Non-cooperative Power Control Algorithm for Wireless Ad Hoc and Sensor Networks," Proc. IEEE Symp. Genetic and Evolutionary Computing (WGEC 08). IEEE Press, Sept. 2007, pp. 181-184, doi:10.1109/WGEC.2008.95.

[15] H. Qin, Y.F. Du and J. Su. "Fault-Tolerant Algorithm for Distributed Primary Detection in Cognitive Radio Networks," Proc. IEEE Symp. Wireless Communications and Trusted Computing (NSWCTC 9th). IEEE Press, Jan. 2009, pp. 353-356, doi:10.1109/NSWCTC.2009.149.

[16] Q. Hu, L.L. ZHAO, Y. Li. "Channel Allocation Algorithm of Cognitive Radio Network Based on Potential Game" Chongqing University of Posts and Telecommunications. Vol. 24, Feb. 2012, pp. 24-28, doi:10.3979/j.issn.1673-825X.2012.01.005.

[17] Q. JIANG, L.K. YANG, L. TANG. "Optimal Power Control for Cognitive Relay Networks Based on Game Theory". Chongqing University of Posts and Telecommunications. Vol. 23, April. 2011, pp. 150-154, doi:10.3979/j.issn.1673-825X.2011.02.005.

[18] N. Yang, H. Tian, P. Hung. "Distributed energy-economical routing algorithm based on game theory for WSN," Journal of 
Electronics\&Information Technology, vol. 30, August. 2008, pp. 1230-1233, doi:CNKI:SUN:DZYX.0.2008-05-052.

[19] R.S. Komali and A.B. MacKenzie. "Distributed Topology Control in Ad-Hoc Networks: A Game Theoretic Perspective," Proc. IEEE Symp. Consumer Communications and Networking Conference (CCNC 06). IEEE Press, Jan. 2006, pp. 563-568, doi:10.1109/CCNC.2006.1593087.

[20] L.R. Hong, Q.H. Meng. "Game-Theoretic Modeling of Joint Topology Control and Power Scheduling for Wireless Heterogeneous Sensor Networks," IEEE Trans, Automation Science and Engineering. Oct. 2009, pp. 610-625, doi:10.1109/TASE.2009.2021321.

[21] C. Taylor and M.A. Nowak. "Transforming the Dilemma," Wiley Online Library. Cambridge, Massachusetts, vol. 61, Oct. 2007, pp. 2281-2292, doi: 10.1111/j.1558-5646.2007.00196.x. 